\title{
Distinct transport mechanism in Candida albicans methylammonium permeases
}

\author{
B. Neuhäuser ${ }^{1}$ (iD
}

Received: 4 March 2020 / Revised: 1 September 2020 / Accepted: 3 September 2020

(C) The Author(s) 2020

\begin{abstract}
It is crucial for the growth and development of an organism whether ammonium is transported across its membranes in a form of $\mathrm{NH}_{4}{ }^{+}$or $\mathrm{NH}_{3}$. The transport of both molecules follows different $\mathrm{pH}$-dependent gradients across membranes and transport of both substrates differentially affects the internal and external $\mathrm{pH}$. As a consequence, they directly influence the physiology and organism development. CaMep2 from Candida albicans shows a dual transceptor function in ammonium transport and sensing. CaMep2 senses low ammonium availability and induces filamentous growth. CaMep1, by contrast, is only active in transport, but not involved in ammonium signaling. Here, both proteins were heterologously expressed in Xenopus laevis oocytes. This study identified electrogenic $\mathrm{NH}_{4}{ }^{+}$transport by CaMep1 and electroneutral $\mathrm{NH}_{3}$ transport by CaMep2, which might be a prerequisite for the induction of pseudohyphal growth.
\end{abstract}

Keywords Candida albicans $\cdot$ Methylammonium permeases · Ammonium · Transport · Transceptor · Filamentous growth . Substrate specificity

\section{Introduction}

While early experiments indicated the need of an active ammonium transport system in organismal membranes (Hackette et al. 1970), transport proteins for ammonium (this term designates to the sum of ammonium $\left[\mathrm{NH}_{4}{ }^{+}\right]$and ammonia $\left[\mathrm{NH}_{3}\right]$ ) were first identified in the 90th in the yeast Saccharomyces cerevisiae (Marini et al. 1994) and the plant Arabidopsis thaliana $(\mathrm{AMT}=A M$ monium Transporter) (Ninnemann et al. 1994). In yeast, they have been identified by complementation assays showing transport of methylammonium. This determined their denomination as Meps (MEthylammonium Permeases). Upon sequence homology and functional analysis, proteins of this AMT/Mep/Rh family have been identified in a great quantity of organisms

Section Editor: Martin Rühl

Electronic supplementary material The online version of this article (https://doi.org/10.1007/s11557-020-01625-0) contains supplementary material, which is available to authorized users.

B. Neuhäuser

benjamin.neuhaeuser@uni-hohenheim.de

1 Institute of Crop Science, Nutritional Crop Physiology, University of Hohenheim, Fruwirthstr. 20, 70593 Stuttgart, Germany throughout all kingdoms of life (Ludewig et al. 2007). All these ammonium transporter proteins share a high sequence homology and a common protein structure with 11 transmembrane helices forming a central pore (Mayer et al. 2006). Three subunits build a functional trimer which is regulated by the interaction of the subunits (Yuan et al. 2007; Loqué et al. 2007; Neuhäuser et al. 2007). Within the substrate transporting pore, the homology between the transporters is especially pronounced (Neuhäuser et al. 2009, 2014). Therefore, it is surprising that different transport mechanisms have been proposed for different protein subfamilies or transporters from different organisms. Molecular dynamic simulations and functional assays suggested ammonia $\left(\mathrm{NH}_{3}\right)$ (Javelle et al. 2008), ammonium ion $\left(\mathrm{NH}_{4}{ }^{+}\right)$(Mayer et al. 2006), net ammonium transport $\left(\mathrm{NH}_{3}+\mathrm{H}^{+}\right)$(Neuhäuser and Ludewig 2014), and ammonium transport coupled to protons $\left(\mathrm{NH}_{4}{ }^{+}+\right.$ $\mathrm{H}^{+}$) (Ortiz-Ramirez et al. 2011). For proteins of the RhRhesus like subfamily, $\mathrm{NH}_{3}$ transport (Ludewig 2006) as well as electrogenic transport has been shown (Nakhoul et al. 2005; Abdulnour-Nakhoul et al. 2016). The substrate specificity of the bacterial model transporter AmtB of Escherichia coli was disputed for a long time but recent lipid-dependent ammonium-induced currents indicated net $\mathrm{NH}_{4}{ }^{+}$transport by EcAmtB (Mirandela et al. 2019). The ammonium transporters from Archaeoglobus fulgidus Amt1 and Amt3 showed electrogenic 
transport (Wacker et al. 2014). Ammonia transport strictly coupled to proton transport is evident in proteins of the plant AMT1 subfamily (Mayer et al. 2006; Neuhäuser et al. 2007). Investigated proteins from the plant AMT2 subfamily showing closer sequence homology to Rh-Rhesus or bacterial AmtB proteins (Ludewig et al. 2007) all mediated electroneutral $\mathrm{NH}_{3}$ transport (Guether et al. 2009; Neuhäuser et al. 2009; Straub et al. 2014). Recently, a dependence of transport on substrate deprotonation from $\mathrm{NH}_{4}{ }^{+}$to $\mathrm{NH}_{3}$ has been shown for many ammonium transporters (Ariz et al. 2018). Therefore, the difference in transport mechanism might only result from a difference in the ratio in which proton transport is coupled to ammonia transport after initial deprotonation.

ScMep2 and its homolog CaMep2 were both shown to be involved in the initiation of invasive growth in low ammonium conditions (Marini et al. 1997; Biswas and Morschhäuser 2005; Neuhäuser et al. 2011). The respective Mep1 and Mep3 proteins were not involved in this mechanism. Yeast Mep proteins seem to be subjected to post-translational regulation by the Npr1 kinase (Dubois and Grenson 1979; Grenson and Dubois 1982; Grenson 1983; Boeckstaens et al. 2007; Neuhäuser et al. 2011). CaMep2 completely lost activity in the $n p r 1$ mutant background. The knockout of Npr1 as well led to a loss of CaMep2-mediated initiation of invasive growth (Neuhäuser et al. 2011). The CaNpr1 and ScNpr1 kinases were proposed to phosphorylate and thereby activate the respective Mep2 proteins at a conserved serine residue in the protein C-termini. A phospho-mimic mutation of this $\mathrm{S}_{457} \mathrm{D}$ was able to activate ScMep2 transport (van den Berg et al. 2016). The respective serine residue in CaMep2 is found at amino acid position 453 .

Even though the nature of the transported substrate and the activity of the different Meps have been proposed to be crucial for yeast growth, the transport mechanisms of Mep transporters have only partially been addressed (Boeckstaens et al. 2008). Transporter activity dependence on the $\mathrm{pH}$ suggested ammonium transport by Mep1 and electroneutral transport by Mep2 (Boeckstaens et al. 2008). A pore-lining twinHis motif is conserved throughout almost all AMT/Mep/Rh proteins, non-electrogenic as well as electrogenic (Ganz et al. 2020). Only some fungal Mep1 proteins exhibit a glutamate residue in the position of the first histidine residue. Exchange of this histidine residue $\left(\mathrm{H}_{194} \mathrm{E}\right)$ in the ScMep2 pore changed the $\mathrm{pH}$ dependence of ScMep2-mediated transport and suggested an associated change in the transport mechanism (Boeckstaens et al. 2008). Recently, electroneutral $\mathrm{NH}_{3}$ transport by ScMep2 was shown to be accompanied with an intracellular $\mathrm{pH}$ modification and was proposed to initiate filamentation signaling (Brito et al. 2020). ScMep1 on the other side mediated electrogenic transport was not able to initiate filamentation and differently affected yeast cytosolic $\mathrm{pH}$ in vivo (Brito et al. 2020). Transport by ScMep2 $\mathrm{H}_{194} \mathrm{E}$ did no further induce filamentous growth and exhibit a cytosolic pH decrease similar to ScMep1 (Brito et al. 2020).

So far, there is no compelling evidence arguing for ammonium transport by CaMep 1 and ammonia transport by CaMep2. This study addresses the important issue of transport mechanisms in the Candida albicans Mep1 and Mep2 proteins by the use of direct uptake and electrophysiology measurements in Xenopus laevis oocytes. It shows net $\mathrm{NH}_{4}^{+}$transport by $\mathrm{CaMep} 1$ and electroneutral $\mathrm{NH}_{3}$ transport by CaMep2 which might be the prerequisite for the induction of pseudohyphal growth by CaMep2 as it was recently proposed for ScMep2 (Brito et al. 2020).

\section{Material and methods}

\section{Constructs}

The coding sequences of CaMep1 (orf19.1614) and CaMep2 (orf19.5672) were amplified from wild-type Candida albicans (SC5314) genomic DNA using primers containing the respective restriction enzyme cut sites for direct cloning into the $\mathrm{pOO} 2$ vector for in vitro transcription (Table S1). PCR products were directly digested and ligated into pOO2; the sequences were validated by Sanger sequencing. Mutagenesis was performed by PCR using primers with the respective miss-matches (Table S1). Mutagenesis was confirmed by Sanger sequencing. RNA for oocyte injection was transcribed in vitro using the mMESSAGE mMACHINETM SP6 Transcription Kit (Thermo Scientific, Bremen, Germany).

\section{Injection of oocytes, electrophysiology, and ${ }^{15} \mathrm{~N}$ uptake}

The electrophysiological methods are described in more detail elsewhere (Mayer and Ludewig 2006). Briefly, oocytes were ordered at Ecocyte Bioscience (Castrop-Rauxel), presorted, and injected with $50 \mathrm{nl}$ of cRNA $(0.8 \mu \mathrm{g} / \mu \mathrm{l})$. Always two different controls were used in parallel. Control oocytes were either not injected or injected with $50 \mathrm{nl}$ of RNAse-free $\mathrm{H}_{2} \mathrm{O}$. Oocytes were kept in ND96 for 4 days at $18{ }^{\circ} \mathrm{C}$ and then placed in a small recording chamber. The recording solution was $110 \mathrm{mM}$ choline chloride, $2 \mathrm{mM} \mathrm{CaCl}, 2 \mathrm{mM} \mathrm{MgCl}_{2}$, and $5 \mathrm{mM}$ MES, $\mathrm{pH}$ adjusted to 5.5 with Tris. Variable ammonium concentrations were added in a form of $\mathrm{NH}_{4} \mathrm{Cl}$ salt. Currents without added ammonium were subtracted at each voltage. The voltage dependence $\delta$ of the $K_{\mathrm{m}}$ was calculated using the following equation: $K_{\mathrm{m}}^{(\delta)}=K_{\mathrm{m}}{ }^{(0 \mathrm{mV})} \mathrm{X} \exp ^{(\delta \mathrm{X} e \mathrm{X}}$ $\mathrm{V} / k \mathrm{XT}$ ), where $\delta$ is the fractional electrical distance, $e$ is the elementary charge, $V$ is the membrane potential, $k$ is the Boltzmann's constant, and $T$ is the absolute temperature. 
For the determination of ammonium uptake, the oocytes were incubated for $30 \mathrm{~min}$ in a recording solution additionally containing $3 \mathrm{mM} 98 \%{ }^{15} \mathrm{~N}$ added as ammonium sulfate salt. The oocytes were washed six times in $\mathrm{H}_{2} \mathrm{O}$ before they were separately put in tin cups for isotope ratio mass spectrometry measurements. Cups were balanced before usage and after freeze-drying of the oocyte. The cup with oocyte was closed, balanced again, and used for ${ }^{15} \mathrm{~N}$ and total $\mathrm{N}$ determination by isotope ratio mass spectrometry. For measurement, an element analyzer (EuroVector, HEKAtech, Wegberg, Germany) coupled to a mass spectrometer (Delta Plus Advantage, Thermo Scientific, Bremen, Germany) was used. $\delta{ }^{15} \mathrm{~N}$ values are calculated vs. air- $\mathrm{N}_{2}$ using the following equation: $\delta$ $\%_{0}=$ (isotope ratio of sample/isotope ratio of air - 1) $\times 1000$. Data are given as means $(n \geq 8) \pm \mathrm{SE}$, capital letters indicate statistical significance $(p<0.05)$ given by one-way ANOVA followed by the post hoc Tukey HSD analysis.

\section{Results}

\section{Net ion transport by CaMep1}

CaMep1 transports ammonium (here $\mathrm{NH}_{4}{ }^{+}$and/or $\mathrm{NH}_{3}$ ) in a largely Npr1-independent manner (Biswas and Morschhäuser 2005; Neuhäuser et al. 2011). Here, heterologous expression of CaMep1 in Xenopus laevis oocytes was used to address the form of substrate transported by CaMep1. The expression of ammonium transporters in Xenopus laevis oocytes has frequently been used for their characterization. Most AMT/Mep/Rh family members analyzed so far were functionally integrated into the oocyte membrane (Neuhäuser et al. 2007, 2009; Guether et al. 2009; Wu et al. 2019; Bindel and Neuhäuser 2019; Brito et al. 2020). The oocytes do not possess endogenous ammonium transporters and provision of ammonium in concentrations of up to $10 \mathrm{mM}$ to water-injected or noninjected controls did not trigger any unspecific currents. This applies to all oocyte batches used during this study. Oocytes expressing CaMep1 yielded large ammoniumdependent currents implying a general net ammonium ion transport function for CaMep1. This transport was concentration-dependent and saturated with increasing ammonium concentrations showing half-maximal transport rates at a $K_{\mathrm{m}}$ of $\sim 0.2 \mathrm{mM}$ (Fig. 1a). The affinity strongly depended on the membrane potential, indicating that like plant AMT1 transporters (Mayer et al. 2006; Neuhäuser et al. 2007), $\mathrm{NH}_{4}{ }^{+}$enters deeply into the membrane electric field before being deprotonated with a fractional electrical distance of $\delta_{\mathrm{NH} 4+}=0.375$. Therefore, $\mathrm{NH}_{4}{ }^{+}$will pass about $37.5 \%$ of the membrane electrical field before it is deprotonated to $\mathrm{NH}_{3}$ (Ganz et al. 2019, 2020).

\section{CaMep2 function in oocytes}

Furthermore, the wild-type CaMep 2 transporter was expressed in Xenopus laevis oocytes. Previous growth and uptake experiments in Candida albicans showed a 20-30\% contribution of CaMep2 to the total ammonium uptake. When CaMep2 was expressed in oocytes, it did not yield any ammonium-induced currents (Fig. 2a, b). This might imply a non-functionality of CaMep2 in oocytes or electroneutral $\mathrm{NH}_{3}$ transport by CaMep2. Protein structures of ScMep2 as well as CaMep2 imply an autoinhibition of the transporters by their cytosolic C-terminal protein end. This autoinhibition was proposed to be disrupted by CaMep2 phosphorylation at $\mathrm{S}_{453}$ (van den Berg et al. 2016). Mimicking phosphorylation by mutation of serine 453 into aspartate should therefore be expected to activate the transporter. Nevertheless, no currents were observed in the $\mathrm{S}_{453} \mathrm{D}$ mutant (Fig. 2a, b). A mutation of the pore-lining histidine 188 to glutamate would resemble the pore profile of the $\mathrm{NH}_{4}{ }^{+}$-transporting CaMep1 in the CaMep2 pore and this was shown to change the transport mechanism of ScMep2 (Boeckstaens et al. 2008; Brito et al. 2020). This mutation did as well not induce ammoniumdependent currents. Despite the potentially activating mutations, no currents could be observed in the CaMep2 variants, even at very negative membrane potential.

\section{CaMep2 is transporting ammonia into oocytes of Xenopus laevis}

To observe a general ammonium/ammonia transport capacity of CaMeps, oocytes expressing CaMep1, the CaMep2 variants as well as water-injected control oocytes not expressing any Mep transporter were subjected to $3 \mathrm{mM}^{15} \mathrm{NH}_{4}{ }^{+}$supplied in the form of ammonium sulfate. Oocytes expressing CaMep2 wild-type showed significant uptake of ${ }^{15} \mathrm{~N}$ compared with water-injected oocytes even though this uptake was not as high as for wild-type CaMep1 (Fig. 3). The proteins with the potentially activating mutations $\mathrm{H}_{188} \mathrm{E}$ or $\mathrm{S}_{453} \mathrm{D}$ rather decreased the CaMep2-mediated uptake of ${ }^{15} \mathrm{~N}$ into the oocytes. Combined with the current measurements, these results suggest electroneutral transport of $\mathrm{NH}_{3}$ by CaMep2.

\section{Discussion}

The exact form of substrate transported by ammonium transporters has been investigated for several AMT/Mep/Rh family members from distinct organisms (Nakhoul et al. 2005; Mayer et al. 2006; Ludewig et al. 2007; Javelle et al. 2008; Neuhäuser et al. 2009; Ortiz-Ramirez et al. 2011; Wacker et al. 2014; Abdulnour-Nakhoul et al. 2016; Mirandela et al. 2019). For Mep proteins of Candida albicans, the transported substrate has been kept an open question (Brito et al. 2020). 

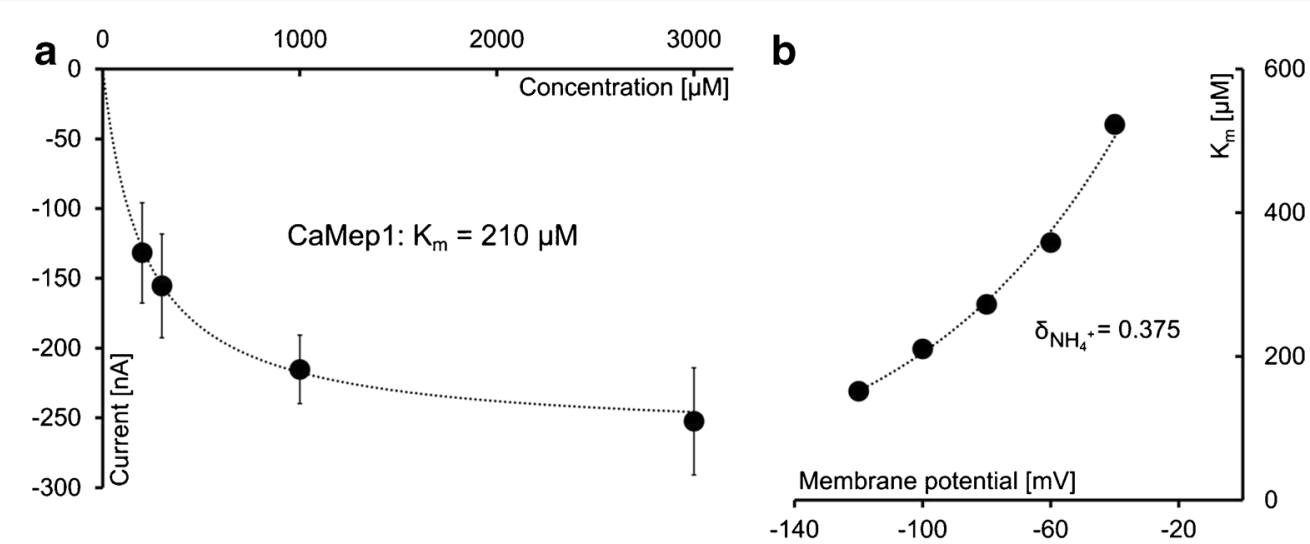

Fig. 1 Ammonium transport kinetics of CaMep1 ammonium transport. To determine the ammonium transport kinetics of CaMep1-expressing oocytes, they were subjected to solutions containing different concentrations of ammonium $\left(0.2 ; 0.3 ; 1 ;\right.$ and $\left.3 \mathrm{mM} \mathrm{NH}_{4}{ }^{+}\right)$. a Currents elicited by ammonium at a membrane potential of $-100 \mathrm{mV}$ were plotted against the

Experiments in Saccharomyces cerevisiae showed different $\mathrm{pH}$ optima for the transport activity of ScMep1 and ScMep2 (Boeckstaens et al. 2008). ScMep2 had a pH optimum in the acidic range around $\mathrm{pH} 4$ (Boeckstaens et al. 2008). ScMep1 showed a transport optimum at $\mathrm{pH}$ 6. The differences in $\mathrm{pH}$ dependence were proposed to be an indication of distinct transport mechanisms in different yeast ammonium transporters (Boeckstaens et al. 2008). Finally, net $\mathrm{NH}_{4}{ }^{+}$transport by ScMep1 and electroneutral $\mathrm{NH}_{3}$ transport by ScMep2 were shown (Brito et al. 2020). Different transport mechanisms have as well been suggested for plant AMT1 and AMT2 subfamily members. While ammonium transport was always accompanied by charge transport in AMT1 transporters (Mayer et al. 2006; Neuhäuser et al. 2007), several plant AMT2 members showed ammonium uptake, but no ammonium-induced currents (Guether et al. 2009; Neuhäuser et al. 2009, 2014; Straub et al. 2014). The electroneutral $\mathrm{NH}_{3}$ transport was strongly $\mathrm{pH}$-dependent as well and, surprisingly, increased at more acidic $\mathrm{pH}$ (Guether et al. 2009). ammonium concentration. In $\mathrm{H}_{2} \mathrm{O}$ injected or not injected control oocytes, addition of ammonium did not induce currents (data not shown). Data are given as means $(n=5) \pm \mathrm{SD}$. b The concentration at which transport was half-maximal $\left(K_{\mathrm{m}}\right)$ is plotted against the membrane potential. Data is calculated from mean values $(n=5)$

As shown for plant AMT1 transporters, ammonium transport by CaMep1 induced strong inward-directed currents in Xenopus laevis oocytes. This transport is saturated with halfmaximal currents at $0.2 \mathrm{mM}$ (Fig. 1a), which is in accordance with transport mediated by ScMep1 (Brito et al. 2020). The affinity of the transporter was strongly dependent on the membrane potential showing a fractional electrical distance of $\delta_{\mathrm{NH} 4+}=0.375$ (Fig. 1b). Assuming that the deprotonation of ammonium in the ammonium transporter pore (Ariz et al. 2018) is the rate-limiting step, the $\delta$ indicates that ammonium passes about $40 \%$ of the membrane electric field before it is deprotonated. Therefore, the ammonium affinity might not be determined by the binding pocket at the pore entrance, but rather by pore internal structures involved in ammonium deprotonation. Two conserved structures, a double Phe-gate and a twin-His motif, are lining AMT/Mep transporter pores. While the Phe-gate of AtAMT1;2 does not seem to be involved in deprotonating the ammonium substrate (Ganz et al. 2019), the twin-His motif of AtAMT1;2 was shown to
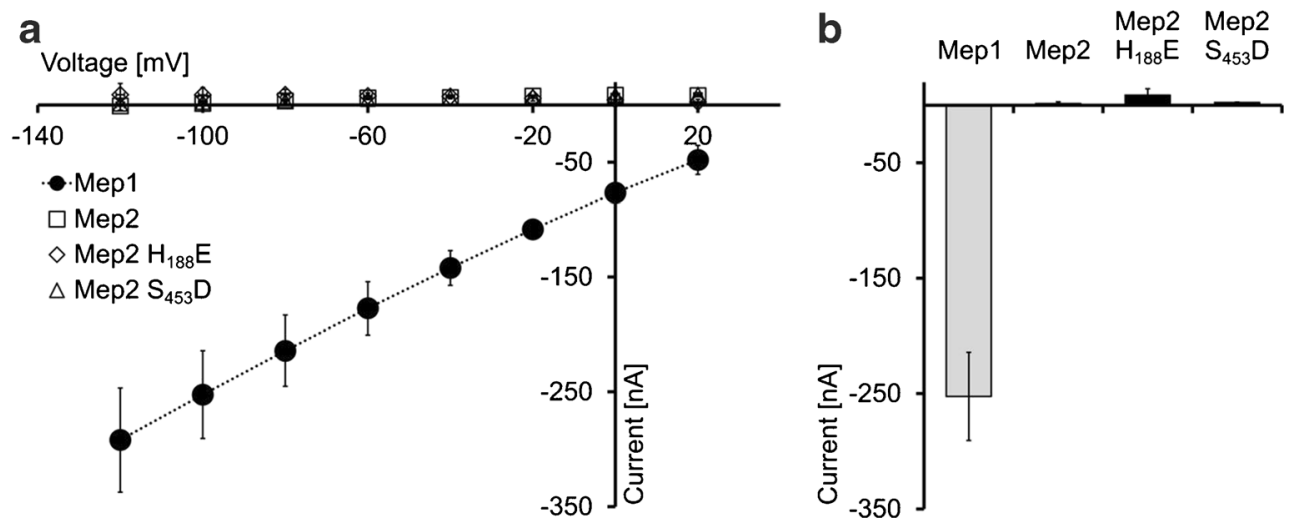

Fig. 2 Currents induced by ammonium. a Current-voltage plot showing currents induced by $3 \mathrm{mM}$ ammonium chloride in Mep1, Mep2, Mep2 $\mathrm{H}_{188} \mathrm{E}$, and Mep2 $\mathrm{S}_{453} \mathrm{D}$ expressing oocytes. Similar to CaMep2expressing oocytes, water injected or not injected controls did not exhibit

ammonium dependent currents (data not shown). Data are given as means $(n=5) \pm \mathrm{SD}$. b Bar chart showing currents at $-100 \mathrm{mV}$ induced by $3 \mathrm{mM}$ ammonium chloride. Data are given as means $(n=5) \pm \mathrm{SD}$ 


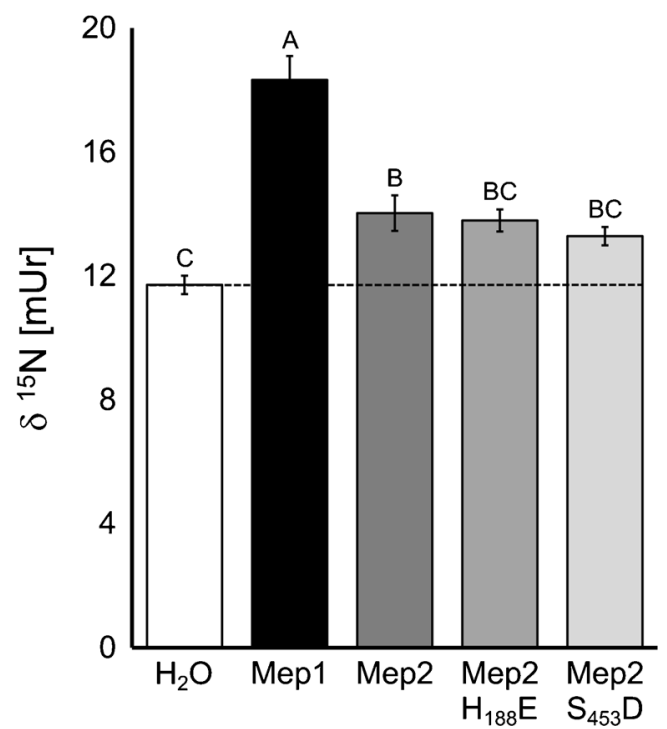

Fig. 3 Changes in $\delta{ }^{15} \mathrm{~N} /{ }^{14} \mathrm{~N}$ by ${ }^{15} \mathrm{NH}_{4}{ }^{+}$uptake into CaMep2-expressing oocytes. Oocytes expressing CaMep1 and the CaMep2 variants as well as $\mathrm{H}_{2} \mathrm{O}$-injected control oocytes (not expressing any Mep protein) were incubated for $30 \mathrm{~min}$ in recording solution containing $3 \mathrm{mM}^{15} \mathrm{NH}_{4}{ }^{+}$ $98 \% . \delta{ }^{15} \mathrm{~N} /{ }^{14} \mathrm{~N}$ values are given as means $(n \geq 8) \pm$ SE; capital letters indicate statistical significance $(p<0.05)$ given by one-way ANOVA with the post hoc Tukey HSD analysis

be essential for deprotonation (Ganz et al. 2020). The position of the twin-His motif in the protein would be in accordance with deprotonation of the substrate after crossing about $40 \%$ of the membrane electric field (Ullmann et al. 2012). As proposed for other AMT/Mep/Rh members, the deprotonation might therefore take place at the position of the twin-His motif in the transporter pore (Ganz et al. 2019, 2020). The first histidine residue in the twin-His motif was not essential for the deprotonation but mutation into a glutamate residue in AtAMT2 decreased the dependence on deprotonation, increased the transport rate, and allowed some bypassing of potassium and possibly ammonium ions (Ganz et al. 2020). This is in line with the observation that, like $\mathrm{NH}_{3}$ transporting plant AMT2s, ScMep2 had a transport optimum in more acidic conditions (Boeckstaens et al. 2008) and mediated electroneutral $\mathrm{NH}_{3}$ transport (Brito et al. 2020). Mutation of the pore-lining histidine 194 to glutamate in ScMep2 changed the $\mathrm{pH}$ optimum to $\mathrm{pH} 6$ similar to the $\mathrm{pH}$ optimum of $\mathrm{ScMep1}$. Higher transport ammonium rates in yeast were shown for the corresponding ScMep2 $\mathrm{H}_{194} \mathrm{E}$ mutant but the instability of the mutant transporter in oocytes precluded its characterization in this system (Brito et al. 2020). This likely was the case for the CaMep2 $\mathrm{H}_{188} \mathrm{E}$ mutant in this study as well again precluding a detailed discussion of this mutant.

Wild-type ScMep2 was clearly stably localized in the oocyte membrane (Brito et al. 2020) as expected for wild-type CaMep2 since both mediated significant uptake of ${ }^{15} \mathrm{NH}_{4}{ }^{+}$into the oocytes. Still, CaMep2 did not induce any $\mathrm{NH}_{4}{ }^{+}$currents when expressed in oocytes (Fig. 2a), as expected if the transported substrate would be $\mathrm{NH}_{3}$. In Candida albicans (Neuhäuser et al. 2011), as well as in Saccharomyces cerevisiae (Boeckstaens et al. 2007), Mep2 function was shown to be dependent on C-terminal phosphorylation by Npr1. Protein structures of CaMep2 and ScMep2 indicate that both proteins are sensitive to an autoinhibition by the non-phosphorylated Cterminus. This autoinhibition is proposed to be relieved by Npr1-dependent phosphorylation (van den Berg et al. 2016). To exclude the general inactivity of CaMep2 by autoinhibition, a $\mathrm{S}_{453} \mathrm{D}$ mutation was introduced to mimic phosphorylation of the C-terminus. While the corresponding ScMep2 $\mathrm{S}_{457} \mathrm{D}$ variant was stable in oocytes but did not increase ScMep2 activity, the $\mathrm{S}_{453} \mathrm{D}$ mutation rather decreased CaMep2 activity (Fig. 3). Relief of the autoinhibition of ScMep2 and CaMep2 therefore does not seem to be needed in Xenopus laevis oocytes. It might be speculated that protein stability is affected by the different membrane composition as shown for EcAmtB (Mirandela et al. 2019) or phosphorylation might be mediated by an oocyte endogenous kinase. Importantly, none of the CaMep2 variants mediated ammonium-induced currents in oocytes (Fig. 2) while the wild-type CaMep2 clearly showed short-term ${ }^{15} \mathrm{~N}$ uptake (Fig. 3).
Fig. 4 Distinct transport mechanism in Candida albicans methylammonium permeases. Schematic model showing net ammonium transport by CaMep 1 in which the proton is cotransported after initial deprotonation. CaMep2 likely depositions the proton to the outside since no charge transfer is accompanying the ${ }^{15} \mathrm{NH}_{3}$ uptake

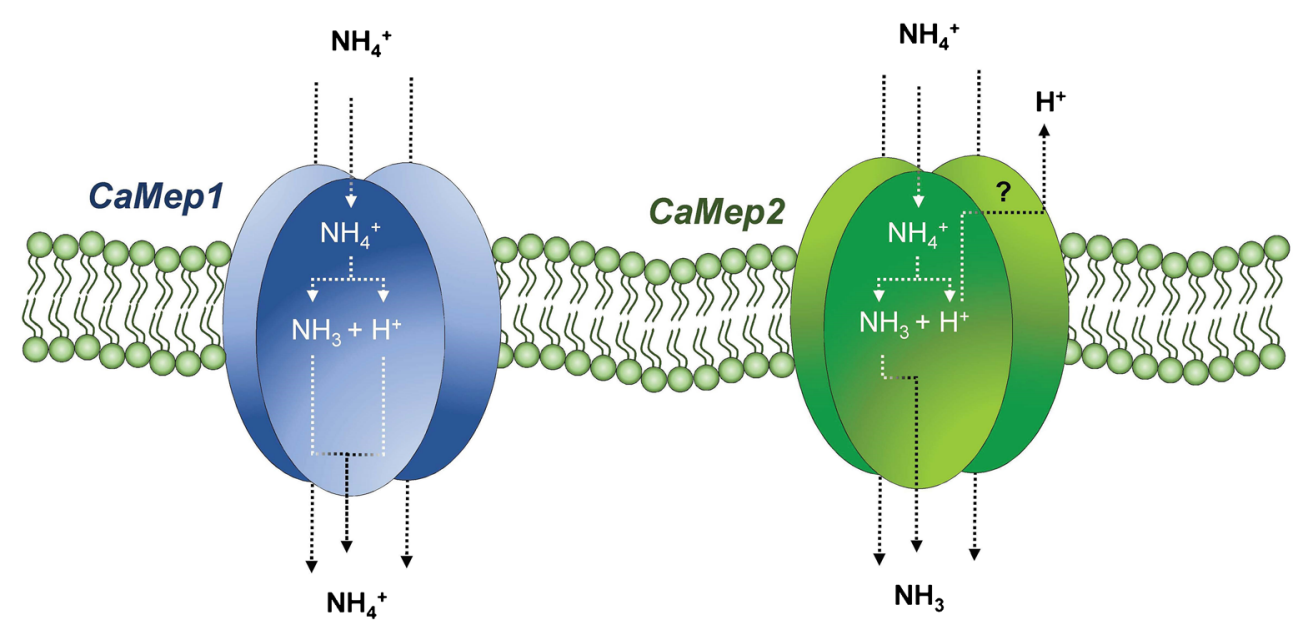


In conclusion, these experiments suggest net $\mathrm{NH}_{4}{ }^{+}$transport (either as $\mathrm{NH}_{4}{ }^{+}$or as $\mathrm{NH}_{3}+\mathrm{H}^{+}$) by CaMep1, but a distinct transport mechanism of electroneutral $\mathrm{NH}_{3}$ conduction by CaMep2 (Fig. 4). Since a general mechanism of ammonium binding and deprotonation during transport was proposed for all AMT/Mep/Rh proteins (Javelle et al. 2008; Ariz et al. 2018), CaMep1 seems to cotransport the proton by a yet unknown pathway. CaMep2 on the other side seems to exclude the proton back outside. Similar results were recently shown for the methylammonium permeases from Saccharomyces cerevisiae. The ScMep2-specific transport mechanism that induces cytosolic alkalinization was suggested to trigger filamentation (Brito et al. 2020). The similarity in Mep transport characteristics between the Candida albicans and Saccharomyces cerevisiae proteins might indicate that the mechanism of inducing filamentous growth by Mep2mediated $\mathrm{NH}_{3}$ transport might as well hold true for Candida albicans.

Acknowledgments I thank Prof. Uwe Ludewig for support and critically reading the manuscript. Furthermore, I thank Prof. Joachim Morschhäuser for introducing me to the fascinating regulation of filamentous growth in Candida albicans and for supplying genomic DNA of Candida albicans.

Author's contributions B.N. planned and performed the experiments and the data analysis and wrote the manuscript.

Funding Open Access funding provided by Projekt DEAL. Work in the laboratory was partially funded by the University of Hohenheim and the DFG.

Data availability All data is part of the manuscript.

\section{Compliance with ethical standards}

Conflict of interest The author declares that there is no conflict of interest.

Open Access This article is licensed under a Creative Commons Attribution 4.0 International License, which permits use, sharing, adaptation, distribution and reproduction in any medium or format, as long as you give appropriate credit to the original author(s) and the source, provide a link to the Creative Commons licence, and indicate if changes were made. The images or other third party material in this article are included in the article's Creative Commons licence, unless indicated otherwise in a credit line to the material. If material is not included in the article's Creative Commons licence and your intended use is not permitted by statutory regulation or exceeds the permitted use, you will need to obtain permission directly from the copyright holder. To view a copy of this licence, visit http://creativecommons.org/licenses/by/4.0/.

\section{References}

Abdulnour-Nakhoul S, Le T, Rabon E et al (2016) Structural determinants of $\mathrm{NH}_{3}$ and $\mathrm{NH}_{4}^{+}$transport by mouse $\mathrm{Rhbg}$, a renal $\mathrm{Rh}$ glycoprotein. Am J Physiol Ren Physiol 311:F1280-F1293. https://doi.org/10.1152/ajprenal.00556.2015

Ariz I, Boeckstaens M, Gouveia C et al (2018) Nitrogen isotope signature evidences ammonium deprotonation as a common transport mechanism for the AMT-Mep-Rh protein superfamily. Sci Adv 4: eaar3599. https://doi.org/10.1126/sciadv.aar3599

Bindel N, Neuhäuser B (2019) Electrophysiological characterization of AtAMT1;4, an extraordinarily high affinity ammonium transporter from Arabidopsis thaliana. bioRxiv 820712. https://doi.org/10. $1101 / 820712$

Biswas K, Morschhäuser J (2005) The Mep2p ammonium permease controls nitrogen starvation-induced filamentous growth in Candida albicans. Mol Microbiol 56:649-669. https://doi.org/10. 1111/j.1365-2958.2005.04576.x

Boeckstaens M, André B, Marini AM (2007) The yeast ammonium transport protein Mep2 and its positive regulator, the Npr1 kinase, play an important role in normal and pseudohyphal growth on various nitrogen media through retrieval of excreted ammonium. Mol Microbiol 64:534-546. https://doi.org/10.1111/j.1365-2958.2007. 05681.x

Boeckstaens M, André B, Marini AM (2008) Distinct transport mechanisms in yeast ammonium transport/sensor proteins of the Mep/ $\mathrm{Amt} / \mathrm{Rh}$ family and impact on filamentation. J Biol Chem 283: 21362-21370. https://doi.org/10.1074/jbc.M801467200

Brito AS, Neuhäuser B, Wintjens R et al (2020) Yeast filamentation signaling is connected to a specific substrate translocation mechanism of the Mep2 transceptor. PLoS Genet 16:e1008634. https://doi. org/10.1371/journal.pgen.1008634

Dubois E, Grenson M (1979) Methylamine/ammonia uptake systems in Saccharomyces cerevisiae: multiplicity and regulation. Mol Gen Genet MGG 175:67-76

Ganz P, Ijato T, Porras-Murillo R et al (2020) A twin histidine motif is the core structure for high-affinity substrate selection in plant ammonium transporters. J Biol Chem. https://doi.org/10.1074/jbc.RA119. 010891

Ganz P, Mink R, Ijato T et al (2019) A pore-occluding phenylalanine gate prevents ion slippage through plant ammonium transporters. Sci Rep 9:16765. https://doi.org/10.1038/s41598-019-53333-9

Grenson M (1983) Study of the positive control of the general amino-acid permease and other ammonia-sensitive uptake systems by the product of the NPR1 gene in the yeast Saccharomyces cerevisiae. Eur J Biochem 133:141-144. https://doi.org/10.1111/j.1432-1033.1983. tb07439.x

Grenson M, Dubois E (1982) Pleiotropic deficiency in nitrogen-uptake systems and derepression of nitrogen-catabolic enzymes in npr-1 mutants of Saccharomyces cerevisiae. Eur J Biochem 121:643647. https://doi.org/10.1111/j.1432-1033.1982.tb05834.x

Guether M, Neuhauser B, Balestrini R et al (2009) A mycorrhizal-specific ammonium transporter from Lotus japonicus acquires nitrogen released by Arbuscular Mycorrhizal fungi. Plant Physiol 150:73-83. https://doi.org/10.1104/pp.109.136390

Hackette SL, Skye GE, Burton C, Segel IH (1970) Characterization of an ammonium transport system in filamentous fungi with methylammonium $-{ }^{14} \mathrm{C}$ as the substrate. J Biol Chem 245:42414250

Javelle A, Lupo D, Ripoche P et al (2008) Substrate binding, deprotonation, and selectivity at the periplasmic entrance of the Escherichia coli ammonia channel AmtB. Proc Natl Acad Sci U S A 105:5040 5045. https://doi.org/10.1073/pnas.0711742105

Loqué D, Lalonde S, Looger LL et al (2007) A cytosolic trans-activation domain essential for ammonium uptake. Nature 446:195-198. https://doi.org/10.1038/nature05579

Ludewig U (2006) Ion transport versus gas conduction: function of AMT/ Rh-type proteins. Transfus Clin Biol 13:111-116. https://doi.org/10. 1016/j.tracli.2006.02.012 
Ludewig U, Neuhäuser B, Dynowski M et al (2007) Molecular mechanisms of ammonium transport and accumulation in plants. FEBS Lett 581:2301-2308. https://doi.org/10.1016/j.febslet.2007.03.034

Marini AM, Soussi-Boudekou S, Vissers S, Andre B (1997) A family of ammonium transporters in Saccharomyces cerevisiae. Mol Cell Biol 17:4282-4293. https://doi.org/10.1128/MCB.17.8.4282

Marini AM, Vissers S, Urrestarazu A et al (1994) Cloning and expression of the MEP1 gene encoding an ammonium transporter in Saccharomyces cerevisiae. EMBO J 13:3456-3463. https://doi. org/10.1002/j.1460-2075.1994.tb06651.x

Mayer M, Dynowski M, Ludewig U (2006) Ammonium ion transport by the AMT/Rh homologue LeAMT1;1. Biochem J 396:431-437. https://doi.org/10.1042/BJ20060051

Mayer M, Ludewig U (2006) Role of AMT1;1 in $\mathrm{NH}_{4}{ }^{+}$acquisition in Arabidopsis thaliana. Plant Biol (Stuttg) 8:522-528

Mirandela GD, Tamburrino G, Hoskisson PA et al (2019) The lipid environment determines the activity of the Escherichia coli ammonium transporter AmtB. FASEB J 33:1989-1999. https://doi.org/10. 1096/fj.201800782R

Nakhoul NL, Dejong H, Abdulnour-Nakhoul SM et al (2005) Characteristics of renal Rhbg as an $\mathrm{NH}_{4}{ }^{(+)}$transporter. Am J Physiol Ren Physiol 288:F170-F181. https://doi.org/10.1152/ ajprenal.00419.2003

Neuhäuser B, Dunkel N, Satheesh SV, Morschhäuser J (2011) Role of the Npr1 kinase in ammonium transport and signaling by the ammonium permease Mep2 in Candida albicans. Eukaryot Cell 10:332342. https://doi.org/10.1128/EC.00293-10

Neuhäuser B, Dynowski M, Ludewig U (2009) Channel-like $\mathrm{NH}_{3}$ flux by ammonium transporter AtAMT2. FEBS Lett 583:2833-2838. https://doi.org/10.1016/j.febslet.2009.07.039

Neuhäuser B, Dynowski M, Ludewig U (2014) Switching substrate specificity of AMT/MEP/Rh proteins. Channels 8:496-502. https://doi. org/10.4161/19336950.2014.967618

Neuhäuser B, Dynowski M, Mayer M, Ludewig U (2007) Regulation of $\mathrm{NH}_{4}{ }^{+}$transport by essential cross talk between AMT monomers through the carboxyl tails. Plant Physiol 143:1651-1659. https:// doi.org/10.1104/pp.106.094243
Neuhäuser B, Ludewig U (2014) Uncoupling of ionic currents from substrate transport in the plant ammonium transporter AtAMT1;2. J Biol Chem 289:11650-11655. https://doi.org/10.1074/jbc.C114. 552802

Ninnemann O, Jauniaux JC, Frommer WB (1994) Identification of a high affinity $\mathrm{NH}_{4}{ }^{+}$transporter from plants. EMBO J. https://doi.org/10. 1002/j.1460-2075.1994.tb06652.x

Ortiz-Ramirez C, Mora SI, Trejo J, Pantoja O (2011) PvAMT1;1, a highly selective ammonium transporter that functions as $\mathrm{H}^{+} / \mathrm{NH}_{4}^{(+)}$ symporter. J Biol Chem 286:31113-31122. https://doi.org/10. 1074/jbc.M111.261693

Straub D, Ludewig U, Neuhäuser B (2014) A nitrogen-dependent switch in the high affinity ammonium transport in Medicago truncatula. Plant Mol Biol 86:485-494. https://doi.org/10.1007/s11103-0140243-4

Ullmann RT, Andrade SLA, Ullmann GM (2012) Thermodynamics of transport through the ammonium transporter Amt-1 investigated with free energy calculations. J Phys Chem B 116:9690-9703. https://doi.org/10.1021/jp305440f

van den Berg B, Chembath A, Jefferies D et al (2016) Structural basis for Mep2 ammonium transceptor activation by phosphorylation. Nat Commun 7:11337. https://doi.org/10.1038/ncomms11337

Wacker T, Garcia-Celma JJ, Lewe P, Andrade SL (2014) Direct observation of electrogenic $\mathrm{NH}_{4}{ }^{+}$transport in ammonium transport (Amt) proteins. Proc Natl Acad Sci U S A:6-11. https://doi.org/10.1073/ pnas. 1406409111

Wu X, Liu T, Zhang Y et al (2019) Ammonium and nitrate regulate $\mathrm{NH}_{4}{ }^{+}$ uptake activity of Arabidopsis ammonium transporter AtAMT1;3 via phosphorylation at multiple C-terminal sites. J Exp Bot 70: 4919-4930. https://doi.org/10.1093/jxb/erz230

Yuan L, Loqué D, Ye F et al (2007) Nitrogen-dependent posttranscriptional regulation of the ammonium transporter AtAMT1;1. Plant Physiol 143:732-744. https://doi.org/10.1104/pp.106.093237

Publisher's note Springer Nature remains neutral with regard to jurisdictional claims in published maps and institutional affiliations. 